\title{
Increased readiness for adaptation and faster alternation rates under binocular rivalry in children
}

\author{
Mariann Hudak 1,2, Patricia Gervan ${ }^{1,2,3}$, Björn Friedrich ${ }^{4}$, Alexander Pastukhov ${ }^{4}$, Jochen Braun ${ }^{4}$ and \\ Ilona Kovacs ${ }^{1,2,3}$ *
}

${ }^{1}$ Hungarian Academy of Sciences, Budapest, Hungary

2 Research Group of Cognitive Science, Budapest University of Technology and Economics, Budapest, Hungary

${ }^{3}$ Department of Cognitive Science, Budapest University of Technology and Economics, Budapest, Hungary

${ }^{4}$ Cognitive Biology, University of Magdeburg, Magdeburg, Germany

\section{Edited by:}

Alexander Maier, Vanderbilt

University, USA

Reviewed by:

Sidney R. Lehky, The Salk Institute, USA

Jan Brascamp, Vanderbilt University, USA

\section{*Correspondence:}

Ilona Kovacs, Department of Cognitive Science, Budapest University of Technology and

Economics, Egry J. u. 1, T 503,

Budapest 1111, Hungary.

e-mail:ikovacs@cogsci.bme.hu
Binocular rivalry in childhood has been poorly investigated in the past. Information is scarce with respect to infancy, and there is a complete lack of data on the development of binocular rivalry beyond the first 5-6 years of age. In this study, we are attempting to fill this gap by investigating the developmental trends in binocular rivalry in pre-puberty. We employ a classic behavioral paradigm with orthogonal gratings, and introduce novel statistical measures (after Pastukhov and Braun) to analyze the data. These novel measures provide a sensitive tool to estimate the impact of the history of perceptual dominance on future alternations. We found that the cumulative history of perceptual alternations has an impact on future percepts, and that this impact is significantly stronger and faster in children than in adults. Assessment of the "cumulative history" and its characteristic time-constant helps us to take a look at the adaptive states of the visual system under multi-stable perception, and brings us closer to establishing a possible developmental scenario of binocular rivalry: a greater and faster relative contribution of neural adaptation is found in children, and this increased readiness for adaption seems to be associated with faster alternation rates.

Keywords: multi-stable perception, binocular rivalry, human development, adaptation, cumulative history, dominance time

\section{INTRODUCTION}

Binocular vision or stereopsis provides precise depth perception by aligning the two eyes' views. Under the eye-specific stimulation of binocular rivalry, the mature visual system enters into a continuous fluctuation between two or more perceptual states, not yielding stereopsis. While cortical binocularity in humans seems to have a relatively abrupt onset (at around 3.5 months) during ontogeny (Braddick et al., 1980; Petrig et al., 1981), driven by experiencedependent mechanisms (Kovacs et al., 2011), little is known about the onset time of binocular rivalry and its further development. Here we review information with respect to the human development of binocular rivalry, and make an attempt to assess its maturity before puberty in a behavioral experiment. We interpret our data in the wider framework of neural adaptation.

The nature of binocular vision of human infants before the occurrence of binocular 3D perception has been debated. This issue was mainly investigated in preferential looking paradigms, employing stimuli that induce binocularly rivalry in adults. Shimojo et al. (1986) found that infants younger than 3.5 months of age preferred to look at the dichoptic (interocularly orthogonal) pattern. However, at an average age of 3.5 months, a sudden shift of preference occurred from the rivalrous pattern to the fusible stimulus. They interpret this result as a preference for a blended stimulus, resulting in a grid-like pattern, which is more complex than the monocularly projected simple lines. However, from the time by which binocular functions have further developed
(3.5 months of age), the two patterns begin to oscillate, which might be aversive for infants. This would account for the shift in the preference for binocularly fusible stimuli, and would suggest that pre-stereoscopic vision blends those images that are rivalrous for adults. However, these results could not be replicated (Brown and Miracle, 2003). Nor did Brown et al. (1999) find any physiological evidence for binocular rivalry using a visually evoked potential paradigm with 5- to 15-month-old infants. They attribute their result to the immaturity of dichoptic suppression.

Even less data have accumulated so far concerning the development of rivalry following infancy. In a study that aimed to compare binocular interactions of children aged 6-14 years to normal and amblyopic adults, it was found that binocular summation decreased with age in a dichoptic visual acuity task (Vedamurthy et al., 2007). The acuity of the dominant eye did not improve significantly in children in the dichoptic viewing condition compared to the monocular condition. In this respect, the performance of children was similar to that of adults. However, they found a significant negative correlation with age in the improvement of acuity of the non-dominant eye in the dichoptic condition compared to the monocular one, indicating that developmental trends in binocular interactions are present after infancy, until at least pre-puberty.

The development of binocular rivalry was investigated in 5- to 6-year-old children (Kovacs and Eisenberg, 2005). They found that children alternated significantly more quickly than adults. Verbal reports of the subjects also indicated that children perceived a 
patchwork of the two images more frequently than adults. On this basis, the conclusion was drawn that the visual system of 5- to 6-year-old children is not sufficiently mature to integrate entire images spatially, thus they experience more piecemeal rivalry than adults. This is in line with their earlier findings in contour integration (Kovacs et al., 1999; Kovacs, 2000) and spatial integration (Káldy and Kovacs, 2003).

Binocular rivalry shares several features with the perception of ambiguous figures, such as the Necker cube. Common features are gamma distribution of the dominance times of each percept, the high inter-subject variability of the frequency of reversals, the significant influence of stimulus properties on reversal rates, or the fact that both can be influenced by the voluntary control of the subject (see Kornmeier and Bach, 2005).

According to the findings reviewed by Leopold and Logothetis (1999), stimulus properties, such as brightness, contrast, and spatial-frequency content can have a significant impact on the balance of dominance and suppression. In addition, high-level properties of the stimuli can also modify dominance periods in multi-stable perception. Such properties include recognizability or semantic content. For instance, if a recognizable figure is inverted, then its perceptual dominance might significantly be altered in both figure/ground stimuli and binocular rivalry.

Voluntary control is another modifying factor of multi-stable perception. The influence of voluntary control of the subject was found to be stronger in the case of ambiguous figures than for binocularly presented rivalrous stimuli (van Ee et al., 2005). Taddei-Ferretti et al. (2008) also point out that the rivalry between the two possible percepts of an ambiguous figure is less automatic than the competition between two different images presented binocularly. An additional common feature of binocular rivalry and ambiguous figures that Taddei-Ferretti et al. (2008) mention is that both are influenced by eye movements (Ellis and Stark, 1978; Sabrin and Kertesz, 1980). Leopold and Logothetis (1999) consider exclusivity, inevitability, and randomness as the three most fundamental common features of multi-stable perception including binocular rivalry and ambiguous figures. Exclusivity means that only one percept is present at one time, while inevitability implies that "perception can never become 'locked' onto a single solution" (p. 261): perceptual hypothesizes are constantly changing concerning the presented stimuli. These attributes are characteristics of both binocular rivalry and ambiguous figures.

Reese and Ford's (1962) pioneering study intended to investigate developmental aspects of ambiguous figure perception. Nursery-school children were shown a series of six pictures of either animals or human faces. Their task was to name each. After that, they were asked to state an expectancy about the next picture. The result was that when they were shown the Bugelski rat-man ambiguous figure, it was easier for them to provide the "animal" interpretation than the "human face" interpretation, which means that the animal interpretation was easier to prime by the previously shown pictures. This might be considered evidence of stimulusdependency even at such an early age. However, 3- to 5-year-old children also show significant performance differences even in this short age range. Doherty and Wimmer (2005) found that 3 year-old children cannot even report both interpretations of such ambiguous images as the duck-rabbit or the man-mouse figures. However, 4-year-old children can easily interpret the ambiguous figures in both ways. Nonetheless, spontaneous reversals occurred only at the age of 5 . The conclusion of this study is that understanding that the perception of the same physical image might reverse is not sufficient for spontaneous reversals to occur.

The foregoing review of the literature indicates that binocular rivalry in childhood has been poorly investigated in the past, and the case is similar concerning the broader sense of bistable or multi-stable perception. Some studies focused on changes in binocular rivalry during adulthood. In these studies, it was found that domination times became longer with age. Jalavisto (1964) for instance, investigated binocular oscillations in the age range of 40-93. It was found that the frequency of oscillation decreased with age in a regular manner, and a total lack of change became prevalent in the oldest age classes. In a more recent study, similar results were obtained (Ukai et al., 2003), in which the alternation rates in three age-groups were compared: 20-34, 35-49, and 5064-year-old subjects were investigated. In line with the results of Jalavisto (1964), they found a prolongation in alternation time as a function of age. Information is still scarce with respect to infancy, and there is a complete lack of data on the development of binocular rivalry beyond the first 5-6 years of life in childhood. In this study, we are attempting to fill this gap by investigating the developmental trends in binocular rivalry in pre-puberty. We employ a classic behavioral paradigm with orthogonal gratings, and introduce novel statistical measures to analyze the data. These novel measures were developed by Pastukhov and Braun (2011), and they provide a sensitive tool to estimate the impact of the history of perceptual dominance on future percept durations. The Pastukhov and Braun (2011) method used here reveals a significant correlation between past perceptual history and future dominance duration, which does not become evident with conventional measures such as sequential correlations of dominance durations (Fox and Herrmann, 1967; Borsellino et al., 1972; Walker, 1975; Lehky, 1995). Assessment of the "cumulative history" and its characteristic time-constant helps us to take a look at the adaptive states of the visual system under multi-stable perception, and brings us closer to establishing a possible developmental scenario for binocular rivalry.

\section{MATERIALS AND METHODS SUBJECTS}

A total of 59 observers participated in the experiment: 9year-olds $(n=23$; mean age $=116.4$ months; $\mathrm{SD}=4.6)$; 12-yearolds $(n=19$; mean age $=151.4$ months; $\mathrm{SD}=4.4) ; 21$-year-olds ( $n=17$; mean age $=249.1$ months; $\mathrm{SD}=27.9)$. All subjects had normal or corrected-to-normal vision and were naive to the purpose of the experiment. Approval of the Budapest University of Technology and Economics (Faculty of Economics and Social Sciences) Ethical Board was obtained. Informed consent was obtained from adult participants or from the parent/caregiver of the child. Observers were not paid for their contribution.

\section{APPARATUS}

Stimuli were generated in real-time and displayed on a $15^{\prime \prime}$ LCD screen, with a spatial resolution of $1366 \times 768$ pixels and a refresh 
rate of $60 \mathrm{~Hz}$. The viewing distance was $60 \mathrm{~cm}$, so that each pixel subtended approximately $0.024^{\circ}$. Anaglyph glasses (red/green) were used for the dichoptic presentation. Responses were obtained by means of a joystick, whose tilt was recorded by a MATLAB program controlling the experiment.

\section{STIMULI}

The binocular rivalry stimulus consisted of two gratings presented dichoptically: radius, $3^{\circ}$; spatial frequency 0.6 cycles/degree; contrast $50 \%$. One grating was tilted leftward by $45^{\circ}$ and the other rightward by $45^{\circ}$. To minimize inter-block effects, tilt for left and right eye was exchanged in every block, and grating-phase was changed by $180^{\circ}$ in every second block (Figure 1A).

\section{PROCEDURE}

Data were collected in a normally lit, quiet room. Initially, subjects were provided anaglyph glasses and invited to view the computer screen with the rivalrous gratings. When asked about their percept, all subjects reported alternating percepts. After this introduction to the stimulus, observers reported their perceptual state continuously using a joystick. The joystick allowed them to report three different percepts (leftward tilt, rightward tilt, and mixed), and in the case of dominant gratings with a particular tilt, the degree of dominance was indicated by the degree of movement. Dominant gratings were indicated by tilting the joystick in the corresponding direction, while subjects were asked to keep the joystick at the center in the case of a mixed percept. The experimental program recorded the joystick tilt at $50 \mathrm{~Hz}$ sampling frequency. The experiment comprised five blocks; each block lasted 5 min. Each block was followed by a 1-min interval, during which subjects were asked to rest (Figure 1B).

\section{STATISTICAL ANALYSIS}

In order to extract perceptual dominance phases from sampled continuous responses, inputs were discretized into left and right percepts using $75 \%$ threshold of maximal joystick tilt (i.e., a percept was labeled as left if joystick was over $-75 \%$ and it was labeled as right if it was over $+75 \%$ of horizontal tilt). Mean dominance time $\left(T_{\text {dom }}\right)$ was computed from the sequence of discrete dominance periods $T_{\mathrm{i}}$.

As a measure of history dependence for multi-stable displays we have used a coefficient of correlation with cumulative history $c_{H}$ (Pastukhov and Braun, 2011), which was computed as follows. Let $S_{x}(t)$ be a record of perceptual experience $x$ as a function of time $t$, defined as unity while percept $x$ dominates, 0.5 during a mixed or patchy percept, and zero when percept $x$ is suppressed. The cumulative history $H_{x}(t)$ computed using a leaky integrator (Tuckwell, 2006) is then given by

$H_{x}(t) \approx \frac{1}{\tau_{H}} \int_{0}^{t} S_{x}\left(t^{\prime}\right) \cdot \mathrm{e}^{\frac{\left(t-t^{\prime}\right)}{\tau_{H}}} d t^{\prime}$

where $x \in\{$ red/green $\}$ denotes a uniform percept and $\tau_{H}$ is a time-constant to be determined empirically. This assumes that the contribution of prior experience decays exponentially, multiple contributions of same percept combine additively, and there is no contribution from competing percept (see Figure 2 for an

A
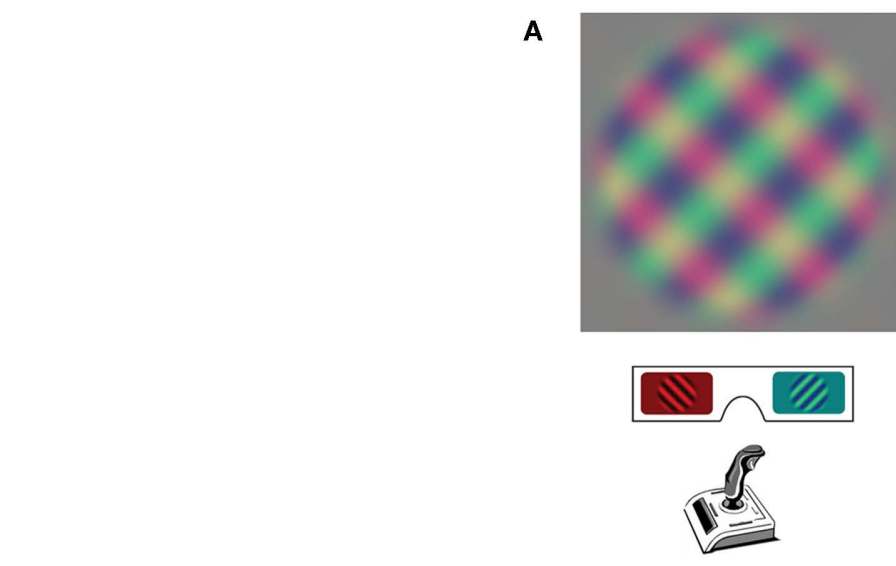

B

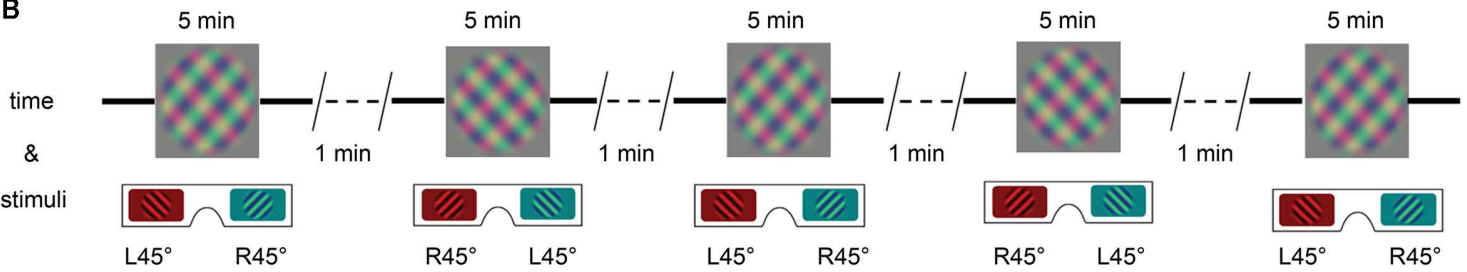

FIGURE 1 | Stimulus and procedure. (A) The orthogonal grating stimuli presented to the two eyes were viewed through anaglyph glasses. Subjects had to indicate the tilt of the perceived grid by tilting the joystick correspondingly. They kept the joystick in the center position in case of mixed percepts. (B) The experiment comprised five blocks of 5-min stimulus presentations, with a 1-min interval following each block. Eye of origin for the orthogonal gratings was exchanged in each block. 


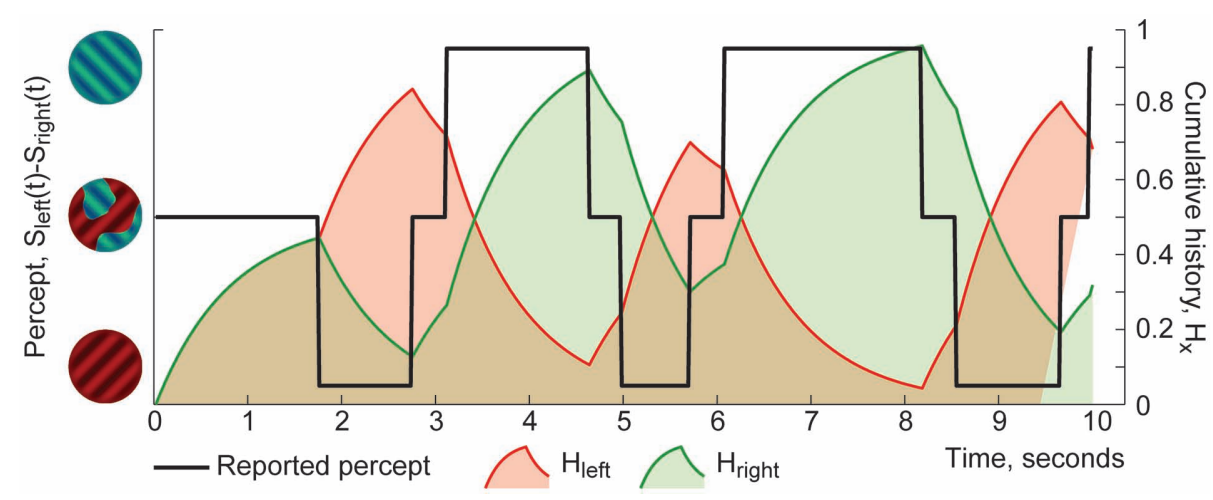

FIGURE 2 | Example of cumulative history traces for series of dominance phases of visual appearances (9 years old). Black trace indicates reported visual appearance ("green/left eye," "red/right eye," or "patchy"). Color traces illustrate hypothetical cumulative histories (correspondingly, green for "green/left eye" percepts and red for "red/right eye"), computed with $\tau_{H}=0.5 \cdot T_{\text {dom }}$ illustration on cumulative history computed from a sequence of perceptual dominance phases).

After computing the cumulative histories $H_{\text {left }}$ and $H_{\text {right }}$ for two alternative percepts from a sequence of dominance periods up to time $t$, we computed linear correlation coefficients with the immediately following dominance period $T_{\mathrm{i}}\left(H_{\text {left }} \times T^{\text {left }}\right.$, $H_{\text {left }} \times T^{\text {right }}, H_{\text {right }} \times T^{\text {left }}$, and $\left.H_{\text {right }} \times T^{\text {right }}\right)$. Specifically, we computed linear correlations between logarithm of its normalized duration and cumulative history for the same and opposite percept, e.g., when left eye is dominant, $S_{\text {left }}(t)=1 ; c_{H}^{\text {same }}=$ $r\left(\ln \left(T_{\mathrm{i}} / T_{\text {dom }}\right), H_{\text {left }}\right)$ and $c_{H}^{\text {diff }}=r\left(\ln \left(T_{\mathrm{i}} / T_{\text {dom }}\right), H_{\text {right }}\right)$. Note that cumulative histories of two competing percepts approach unity $\left(H_{\text {left }}+H_{\text {right }} \approx 1\right)$ only in the absence of "patchy" percepts, we have used both to compute an average absolute correlation:

$c_{H}=\frac{\left|c_{H}^{\text {diff }}\right|+\left|c_{H}^{\text {same }}\right|}{2}$

To determine the characteristic time-constant $\left(\tau_{H}\right)$, we computed average absolute correlations for values of $\tau$ ranging from 0.01 to $60 \mathrm{~s}$. The maximal correlation obtained was taken as the value of $c_{H}$, and the $\tau$ yielding this maximal correlation was taken as the value of $\tau_{H}$. In sum, $c_{H}$ stands for the measure of adaptation taking into account the entire stimulus presentation up to time $t$, while $\tau_{H}$ indicates how fast the adaptation is built up. Note that for small $\tau_{H}$ cumulative history assumes intermediate values only after one or more short dominance periods. The higher the $\tau_{H}$ value is, the slower the subject adapts to each percept; while a higher $c_{H}$ value indicates a larger extent of adaptation.

After computing the above-mentioned variables, outliers were excluded from each group. The criterion for exclusion was identical for each group. The SD of each subject was computed for each variable across the blocks. The mean SD of each group was also computed from the individual SDs. The individual SDs here indicate the reliability of the perceptual reports of the particular subject: the responses of subjects who show a high SD among a given observable, can be considered as inconsistent, which might be due to either lack of attention or fatigue. Therefore, subjects, whose SD along any of the investigated variables approached the
4 SD distance from the average SD of the group, were excluded from the analysis. This criterion was re-checked following each exclusion. A total of eight subjects were excluded.

After removing extreme outliers, independent sample $t$-tests were conducted between all groups for all the five variables, and correlations were computed between age-groups and observables.

\section{RESULTS}

The $t$-test yielded a marginally significant difference in average dominance times $\left(T_{\text {dom }}\right)$ between 9-year-olds and adults (for means and $t$-values see Figure 3A). Each percept tends to persist for a longer period in adults than in 9-year-old children (Figure 3B). There was no significant difference between 9- and 12 -year-olds, and between 12 -year-olds and adults. However, the developmental trend in Figure 3B seems to be clear: dominance times increase with age. The same tendency was found earlier in 5- to 6-year-olds as compared with adults (Kovacs and Eisenberg, 2005).

The $c_{H}$ value is significantly higher in 9-year-olds than adults, i.e., the length of the subsequent dominance period of a particular percept shows a higher correlation with the previous dominance time ratio of the other percept in 9-year-olds than in adults (Figures 3A,C). This means that 9-year-olds and adults show a significant difference in their extent of adaptation to each percept. There was no significant difference between 9- and 12-year-olds, and between 12-year-olds and adults.

The time-constant of the build-up of the adaptation $\left(\tau_{H}\right)$ produced significant differences both between 9-year-olds and adults as well as 12-year-olds and adults (Figures 3A,D). There was no significant difference between 9- and 12-year-olds. The $\tau_{H}$ value of adults is significantly higher than that of 9- and 12-year-old children, showing that the build-up of adaptation is slower in adults.

These differences were also indicated by correlations between age-groups and the observables (see Table $\mathbf{1}$ ).

\section{DISCUSSION}

Our results indicate that 9-year-old children are not exactly adultlike in terms of alternation rate which is a conventional measure 
A

\begin{tabular}{|c|c|c|c|c|c|c|c|}
\hline & & \multicolumn{2}{|c|}{$\begin{array}{l}9 \text { vs } 12 \\
\text { years }\end{array}$} & \multicolumn{2}{|c|}{$\begin{array}{c}9 \text { vs } 21 \\
\text { years }\end{array}$} & \multicolumn{2}{|c|}{$\begin{array}{c}12 \text { vs } 21 \\
\text { years }\end{array}$} \\
\hline \multirow{4}{*}{$\begin{array}{l}\mathrm{T}_{\text {dom }} \\
\text { (sec) }\end{array}$} & Mean & 1,954 & 2,274 & 1,954 & 2,834 & 2,274 & 2,834 \\
\hline & $\mathrm{SD}$ & 0,527 & 0,821 & 0,527 & 1,631 & 0,821 & 1,631 \\
\hline & $\mathrm{t}$ & \multicolumn{2}{|c|}{$-1,398$} & \multicolumn{2}{|c|}{$-2,070$} & \multicolumn{2}{|c|}{$-1,226$} \\
\hline & $p$ & \multicolumn{2}{|c|}{0,171} & \multicolumn{2}{|c|}{0,053} & \multicolumn{2}{|c|}{0,233} \\
\hline \multirow{4}{*}{$\tau_{H}$} & Mean & 0,364 & 0,337 & 0,364 & 0,257 & 0,337 & 0,257 \\
\hline & $S D$ & 0,165 & 0,212 & 0,165 & 0,097 & 0,212 & 0,097 \\
\hline & $\mathrm{t}$ & \multicolumn{2}{|c|}{0,410} & \multicolumn{2}{|c|}{2,277} & \multicolumn{2}{|c|}{1,386} \\
\hline & $p$ & \multicolumn{2}{|c|}{0,684} & \multicolumn{2}{|c|}{0,029 * } & \multicolumn{2}{|c|}{0,176} \\
\hline \multirow{4}{*}{$\mathrm{C}_{\mathrm{H}}$} & Mean & 0,146 & 0,142 & 0,146 & 0,429 & 0,142 & 0,429 \\
\hline & $\mathrm{SD}$ & 0,158 & 0,150 & 0,158 & 0,392 & 0,150 & 0,392 \\
\hline & $\mathrm{t}$ & \multicolumn{2}{|c|}{0,071} & \multicolumn{2}{|c|}{$-2,710$} & \multicolumn{2}{|c|}{$-2,734$} \\
\hline & $p$ & \multicolumn{2}{|c|}{0,944} & \multicolumn{2}{|c|}{$0,013^{*}$} & \multicolumn{2}{|c|}{$0,013^{*}$} \\
\hline
\end{tabular}

C

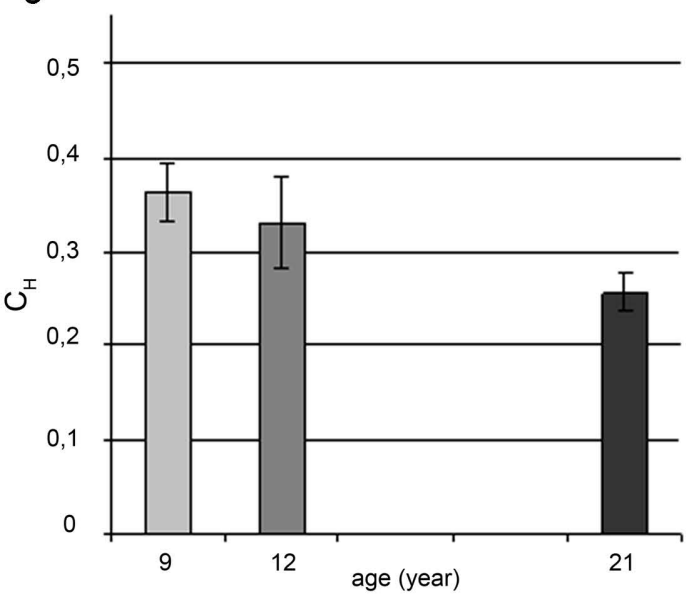

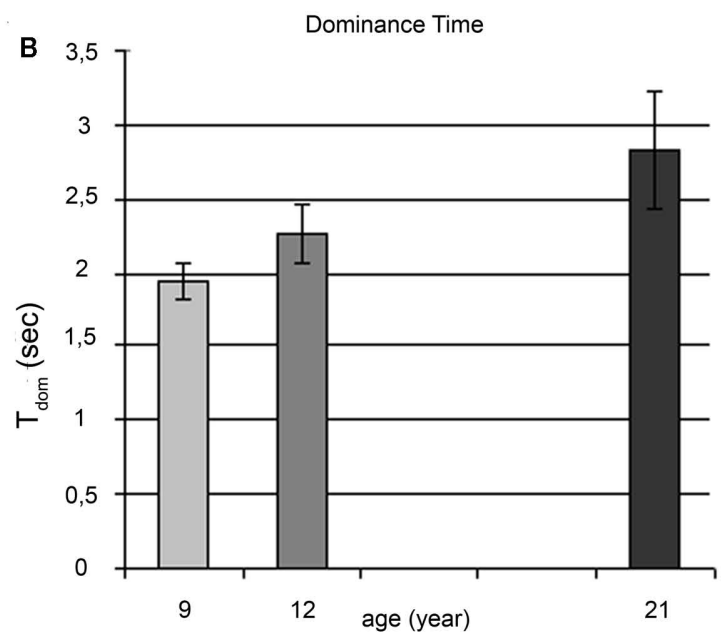

D

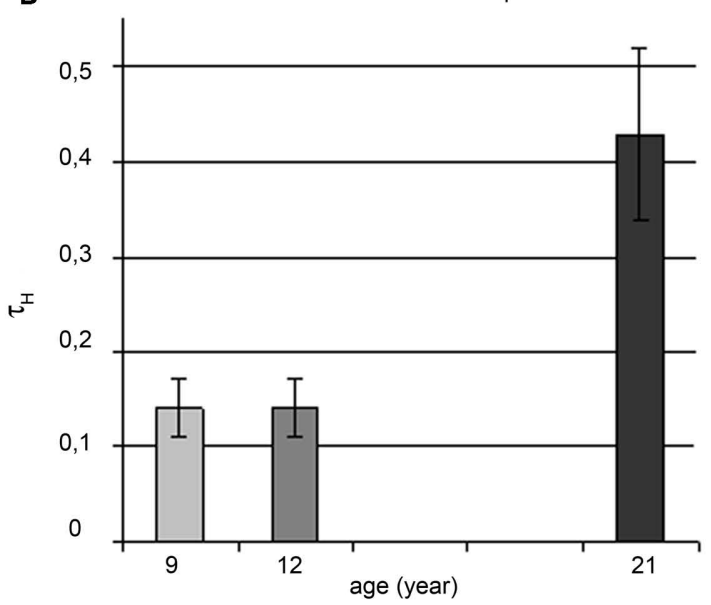

how fast the adaptation is built up. (B) Dominance times $\left(T_{\text {dom }}\right)$ within age-groups. (C) Cumulative history $\left(C_{H}\right)$ within age-groups. (D) Time-constant of adaptation within age-groups. Error bars indicate SE.
FIGURE 3 | Results. (A) Means, SDs, $t-$, and $p$-Values for each variable. $T_{\text {dom }}$ is mean dominance time; Coefficient of correlation with cumulative history $c_{H}$ is a measure of history dependence; Characteristic time-constant $\tau_{H}$ indicates
Table 1 | Correlations between age-groups and the investigated observables.

\begin{tabular}{lllc}
\hline Correlations & $\boldsymbol{T}_{\text {dom }}$ & $\boldsymbol{\tau}_{\boldsymbol{H}}$ & $\boldsymbol{C}_{\boldsymbol{H}}$ \\
\hline AGE-GROUP & & & \\
Pearson correlation & 0.329 & 0.457 & -0.273 \\
Sig. (two-tailed) & 0.018 & 0.001 & 0.053 \\
$N$ & 51 & 51 & 51
\end{tabular}

of binocular rivalry. Children seem to have shorter average dominance times than adults. This is consistent with an earlier study by Kovacs and Eisenberg (2005) that showed that 5- to 6-yearold children are alternating very quickly. Our findings are also in line with the results of Jalavisto (1964) and Ukai et al. (2003), who found that alternation rate decreased with age in adulthood. Although the developmental curve is not complete yet, and there are several further age-groups to be tested, it can be concluded that the development of binocular rivalry, as measured by its most salient feature, is not complete by the end of the first decade in life. That draws a conspicuously slow developmental trajectory which is not yet supported by explanatory anatomical or physiological data.

In addition, we have applied two novel measures of the effect of neural adaptation, recently suggested by Pastukhov and Braun (2011). The first such measure $\left(c_{H}\right)$ was the correlation between dominance times and accumulated prior dominance history and the second $\left(t_{H}\right)$ was the effective time-constant of this accumulation. To appreciate the import (and limitations) of these measures, one has to consider that perceptual reversals may have several contributing causes (Wolfe, 1984; Nawrot and Blake, 1989; Petersik, 2002; van Ee, 2009; Alais et al., 2010; Kang and Blake, 2010; Pastukhov and Braun, 2011).

Firstly, neural adaptation of the dominant representation is thought to progressively destabilize the dominant percept by both the adaptation of the dominant percept, and the recovery from 
adaptation of the suppressed percept. Secondly, spontaneous activity fluctuations in perceptual representations as well as external transients such as eye movements or eye blinks curtail the duration of dominance periods. Thirdly, internal transients such as shifts in attention or in other volitional processes may trigger reversals. The measure $c_{H}$ is a correlative measure and estimates only the relative contribution of neural adaptation to reversal timing, that is, relative to all other possible factors. We emphasize that it should not be taken to estimate the absolute strength of neural adaptation.

Specifically, our finding that dominance durations are more correlated with prior history in children than in adults, implies simply a greater relative contribution of neural adaptation. This could either be because adaptation is more pronounced, or because other factors (e.g., neural noise, attention shifts) are less pronounced in children. Our observations that shorter dominance phase duration in children are accompanied by shorter timeconstants of reconstructed neural adaptation are consistent with predictions of models of multi-stable perception (Wilson, 2007; Shpiro et al., 2009), where mean dominance duration is directly

\section{REFERENCES}

Alais, D., Cass, J., O'Shea, R. P., and Blake, R. (2010). Visual sensitivity underlying changes in visual consciousness. Curr. Biol. 20, 1362-1367.

Borsellino, A., De Marco, A., Allazetta, A., Rinesi, S., and Bartolini, B. (1972). Reversal time distribution in the perception of visual ambiguous stimuli. Kybernetik 10, 139-144.

Braddick, O., Atkinson, J., Julesz, B., Kropfl, W., Bodis-Wollner, I., and Raab, E. (1980). Cortical binocularity in infants. Nature 288, 363-365.

Brown, A. M., and Miracle, J. A. (2003). Early binocular vision in human infants: limitations. Vision Res. 43, 1563-1574.

Brown, R. J., Candy, T. R., and Norcia, A. M. (1999). Development of rivalry and dichoptic masking in human infants. Invest. Ophthalmol. Vis. Sci. 40, 3324-3333.

Chong, S. C., Tadin, D., and Blake, R. (2005). Endogenous attention prolongs dominance durations in binocular rivalry. J. Vis. 5, 1004-1012.

Doherty, M. J., and Wimmer, M. C. (2005). Children's understanding of ambiguous figures: which cognitive developments are necessary to experience reversal? Cogn. Dev. 20, 407-421.

Ellis, S. R., and Stark, L. (1978). Eye movements during the viewing of Necker cubes. Perception 7, 575-581.

Fox, R., and Herrmann, J. (1967). Stochastic properties of binocular rivalry alternations. Percept. Psychophys. 2, 432-446.
Hancock, S., and Andrews, T. J. (2007). The role of voluntary and involuntary attention in selecting perceptual dominance during binocular rivalry. Perception 36, 288-298.

Jalavisto, E. (1964). The phenomenon of retinal rivalry in the aged. Gerontologia 9, 1-8.

Káldy, Z., and Kovacs, I. (2003). Visual context integration is not fully developed in 4-year-old children. Perception 32, 657-666.

Kang, M.-S., and Blake, R. (2010). What causes alternations in dominance during binocular rivalry? Atten. Percept. Psychophys. 72, 179-186.

Kornmeier, J., and Bach, M. (2005). The Necker cube - an ambiguous figure disambiguated in early visual processing. Vision Res. 45, 955-960.

Kovacs, E., Mikó-Baráth, K., Markó, K., Hollódy, B., and Török, G. J. (2011). Ready to experience: binocular function is turned on earlier in preterm infants. Society for Neuroscience Annual Meeting, Washington.

Kovacs, I. (2000). Human development of perceptual organization. Vision Res. 40, 1301-1310.

Kovacs, I., and Eisenberg, M. (2005). "Human development of binocular rivalry," in Binocular Rivalry, eds D. Alais and R. Blake (Cambridge: MIT Press), 101-116.

Kovacs, I., Kozma, P., Fehér, Á., and Benedek, G. (1999). Late maturation of visual spatial integration in humans. Proc. Natl. Acad. Sci. U.S.A. 96, 12204-12209.

proportional to the adaptation time-constant. A related possibility is that, due to the generally shorter dominance times of children, stochastic factors such as neural noise or attention shifts simply have fewer opportunities for triggering a perceptual reversal. Voluntary control over binocular rivalry is limited (Chong et al., 2005; Hancock and Andrews, 2007) and in fact less than for other bistable displays (Meng and Tong, 2004). Rivalrous displays undergo perceptual reversals even when attention is diverted (Lee et al., 2007). Nevertheless, we cannot rule out the possibility that our findings reflect differences in attentional characteristics between children and adults.

Although our study provides the first articulate view on the human developmental trajectory of binocular rivalry, more age-groups, and the underlying factors behind the protracted developmental curve need to be further investigated.

\section{ACKNOWLEDGMENTS}

We thank János Geier and Anett Szabó for helpful discussions on the statistics. This work was supported by TÁMOP - 4.2.2.B-10/12010-0009.

Lee, S.-H., Blake, R., and Heeger, D. J. (2007). Hierarchy of cortical responses underlying binocular rivalry. Nat. Neurosci. 10 1048-1054.

Lehky, S. R. (1995). Binocular rivalry is not chaotic. Proc. Biol. Sci. 259, 71-76.

Leopold, D. A., and Logothetis, N. K. (1999). Multistable phenomena: changing views in perception. Trends Cogn. Sci. (Regul. Ed.) 3, 254-264.

Meng, M., and Tong, F. (2004). Can attention selectively bias bistable perception? Differences between binocular rivalry and ambiguous figures. J. Vis. 4, 539-551.

Nawrot, M., and Blake, R. (1989). Neural integration of information specifying structure from stereopsis and motion. Science 244 716-718.

Pastukhov, A., and Braun, J. (2011). Cumulative history quantifies the role of neural adaptation in multistable perception. J. Vis. 1, 12.

Petersik, J. T. (2002). Buildup and decay of a three-dimensional rotational aftereffect obtained with a three dimensional figure. Perception 31, 825-836.

Petrig, B., Julesz, B., Kropfl, W., Baumgartner, G., and Anliker, M. (1981). Development of stereopsis and cortical binocularity in human infants: electro-physiological evidence. Science 213, 1402-1404.

Reese, H. W., and Ford, L. R. Jr. (1962). Expectancy and perception of an ambiguous figure in preschool children. J. Verbal Learn. Verbal Behav. 1, 188-191.
Sabrin, H. W., and Kertesz, A. E. (1980). Microsaccadic eye movements and binocular rivalry. Percept. Psychophys. 28, 150-154.

Shimojo, S., Bauer, J. Jr., O’Connell, K. M., and Held, R. (1986). Pre-stereoptic binocular vision in infants. Vis. Res. 26, 501-510.

Shpiro, A., Moreno-Bote, R., Rubin, N., and Rinzel, J. (2009). Balance between noise and adaptation in competition models of perceptual bistability. J. Comput. Neurosci. 27, 37-54.

Taddei-Ferretti, C., Radilovac, J., Musioa, C., Santilloa, S., Cibellib, E., Cotugnoa, A., and Radilc, T. (2008). The effects of pattern shape, subliminal stimulation, and voluntary control on multistable visual perception. Brain Res. 1225, 163-170.

Tuckwell, H. C. (2006). Introduction to theoretical neurobiology: linear cable theory and dendritic structure. Cambridge: Cambridge University Press, 1.

Ukai, K., Ando, H., and Kuze, J. (2003). Binocular rivalry alternation rate declines with age. Percept. Mot. Skills 97, 393-397.

van Ee, R. (2009). Stochastic variations in sensory awareness are driven by noisy neuronal adaptation: evidence from serial correlations in perceptual bistability. J. Opt. Soc. Am. A Opt. Image Sci. Vis. 26, 2612-2622.

van Ee, R., van Dam, L. C. J., and Brouwer, G. J. (2005). Voluntary control and the dynamics of perceptual bi-stability. Vision Res. 45, 41-55. 
Vedamurthy, I., Suttle, C. M., Alexander, J., and Asper, R. J. (2007). Interocular interactions during acuity measurement in children and adults, and in adults with amblyopia. Vision Res. 47, 179-188.

Walker, P. (1975). Stochastic properties of binocular-rivalry alternations. Percept. Psychophys. 18, 467-473.

Wilson, H. R. (2007). Minimal physiological conditions for binocular rivalry and rivalry memory. Vision Res. 47, 2741-2750.

Wolfe, J. M. (1984). Reversing ocular dominance and suppression in a single flash. Vision Res. 24, 471-488.

Conflict of Interest Statement: The authors declare that the research was conducted in the absence of any commercial or financial relationships that could be construed as a potential conflict of interest.

Received: 06 July 2011; accepted: 17 October 2011; published online: 04 November 2011.

Citation: Hudak M, Gervan P, Friedrich B, Pastukhov A, Braun J and Kovacs I (2011) Increased readiness for adaptation and faster alternation rates under binocular rivalry in children.
Front. Hum. Neurosci. 5:128. doi: 10.3389/fnhum.2011.00128

Copyright (c) 2011 Hudak, Gervan, Friedrich, Pastukhov, Braun and Kovacs. This is an open-access article subject to a non-exclusive license between the authors and Frontiers Media SA, which permits use, distribution and reproduction in other forums, provided the original authors and source are credited and other Frontiers conditions are complied with. 